\title{
Temperature dependent emissivity of different stainless steel textiles in the infrared range.
}

\author{
M.C. Larciprete, ${ }^{1}$ Y.S. Gloy, ${ }^{2}$ R. Li Voti, ${ }^{1}$ G. Cesarini, ${ }^{1}{ }^{\text {G.Leahu }},{ }^{1}$ M. Bertolotti, ${ }^{1}$ C. Sibilia ${ }^{1}$ \\ 1) Dipartimento di Scienze di Base ed Applicate per l'Ingegneria. Sapienza Università di Roma, Via A. \\ Scarpa 16, 00161 Roma, Italy \\ 2) Institut fur Textiletechnik der RWTH, Aachen University, Otto-Blumenthal-Str. 1, 52074 Aachen \\ Germany.
}

Corresponding author: mariacristina.larciprete@uniroma1.it

\begin{abstract}
We experimentally investigated the infrared properties of a set of steel textiles, prepared using different type of fabrics. Infrared emission of the textiles was characterized in the mid-infrared range, i.e. $8 \div 14$ microns, by observing their temperature evolution under heating regime with a focal plane array (FPA) infrared camera. Standard test method for measuring and compensating emissivity using infrared imaging radiometers was applied to the set of metallic textiles. The obtained experimental results allowed to retrievethe infrared emissivity at different applied temperatures. Althoughtheir infrared emission show some differences depending on the specific fabric, all the investigated textiles composed of steel yarns appear to be suitable for thermal shielding applications. Finally, the measured data were interpreted by means of the finite-difference time-domain (FDTD) numerical simulations as well as using Plank's theory of black-body radiation.
\end{abstract}




\section{INTRODUCTION}

The term infrared signature generically describes how objects appear to infrared sensors and represents a critical property, in particular under night vision conditions [1]. The infrared signature of a given object is determined by several factors, as its shape and size, its temperature and its emissivity, as well as external conditions (i.e., illumination, surface quality, environment, etc.). One of the most challenging tasks is to hide an object at a given temperature by altering its infrared signature [2]. By definition, the IR spectrum is very wide, spanning the range from $0.77 \mu \mathrm{m}$ to $100 \mu \mathrm{m}$, i.e. from red-light to Terahertz radiation. However, only two atmospheric windows allow efficient IR transmittance, i.e. $3 \div 5$ and $8 \div 14 \mu \mathrm{m}$, known as mid wavelength IR windows (MWIR). Outside these windows, attenuation of IR radiation is strong, due to the role of $\mathrm{CO}_{2}$ and $\mathrm{H}_{2} \mathrm{O}$ vapor in both absorption and scattering phenomena [3]. For this reason the reduction of the infrared signature results an important topic within the above mentioned IR windows.

The most efficient approach to reduce the thermal signature is to reduce the IR emission of a given object. Selective thermal emission can be achieved by controlling material spectral absorbance or, equivalently, by managing material emissivity. In the last years, several works have been made within this frame, using both periodical [4-8] and randomly oriented structures [9-10], in the seek for configurations that avoids complicated preparation steps and high costs. Very recently, several types of metallic textiles have been realized and successfully employed for the shielding of electromagnetic radiation in the visible and near IR regime (up to $1100 \mathrm{~nm}$ ) [11] as well as in the radio frequency range [12], including woven fabrics containing stainless steel wires [13] and stainless steel composite fabric [14]. Considering thermal radiation shielding, some aluminum coatings and conductive metalized fibers have been successfully investigated in the $(20 \div 40)^{\circ} \mathrm{C}$ temperature range [15] as well as some multifunctional metal composite fabrics [16]. 
In the present work we show the thermal shielding properties in the mid-wavelength IR regime of several textile structures composed of steel yarns, for IR signature reduction. The investigated textile structures differ by fabrics (woven, knitted and non-woven) and metal content, all of them including conductive yarns of stainless steel which determine the low absorption/emission properties within the investigated mid-infrared range. These structures were characterized through air permeability tests. Furthermore, a focal plane array (FPA) infrared camera $(8 \div 14 \mu \mathrm{m})$ was employed to investigate samples emissivity at increasing temperature. The infrared thermography characterization, combined with thermocouple measurements, allowed to retrieve an important parameter such as sample IR emissivity. Finally, in order to give an interpretation of experimental results, the obtained data were interpreted by means of the finite-difference time-domain (FDTD) numerical simulations as well as using Plank's theory of black-body radiation.

\section{II.SAMPLE PREPARATION}

The four different textile samples that we investigated are reported in Table I. All samples were produced by Bekaert/Bekintex NV (Wetteren, Belgium) and are made of stainless steel fibers which can continuously withstand temperatures up to $700^{\circ} \mathrm{C}$. In Figure 1 we show the digital images obtained by optical microscopy from the different investigated fabrics, respectively. In order to compare the different metallic fabrics, same magnification was employed for all samples. The first two samples (woven-1 and woven-2) display woven structure (Figure 1a and 1b), which ischaracterized by an orthogonal crossing of so called warp and weft yarns. Their thickness was measured according to the ISO 5084 standard (for determination of thickness of textiles and textile products) and was found to be $0.5 \mathrm{~mm}$. The third sample (knitted) display knitted texture (Figure 1c). Knitted textiles consist of a number of consecutive rows of loops. Due to their structure, these textiles are generally more elastic with respect to other textile structures. Finally, non-woven fabrics (felts) are broadly defined as sheet or web structures bonded 
together by entangling fibers or filaments either mechanically, thermally, or chemically. Anon-woven sample is displayed in Figure 1d andis $1.2 \mathrm{~mm}$ thick.

Air permeability test, according to ASTM D 737-96 standard (Standard Test Method for Air Permeability of Textile Fabrics),was performed in order to characterize the metallic textile samples. This method covers the measurement of the rate of air flow passing perpendicularly through a known area under a prescribed air pressure differential between the two surfaces of a material. It is generally employed for the characterization of textile fabrics and is applicable to most fabrics including woven fabrics, knitted fabrics, layered fabrics, and pile fabrics. The value measured for three of the investigated samples, expressed in units as $1 / \mathrm{dm}^{2} / \mathrm{min}$, are reported in Table I. Because the investigated non-woven sample is made with a stainless steel woven scrim sandwiched between two needle felts, air filtration is usually low, thus the air permeability was not measured for this type of fabric.Air permeability is a significant factor for the characterization of such metallic textiles because it strongly depends on the textile construction like the amount of warp and weft yarn per $\mathrm{cm}^{2}$. Having more yarns per $\mathrm{cm}^{2}$ also implies more metal content per $\mathrm{cm}^{2}$. In other words, air permeability gives an indirect information about the metal content in the fabric: lower values of air permeability, correspond to higher metal content in the textile, thus strengthening the thermal shielding performance.

Samples were further characterized using both Scanning Electron Microscopy and Energy Dispersive X-ray Spectroscopy (SEM/EDS analysis). Quantitatively, the weight percent (wt\%) of elements such as oxygen, iron, chromium and nickel was measured with mapping scanning EDS analysis and the obtained values are reported in Table II. Although the four samples display almost same iron amount, there are some differences for the other elements. Typically, chromium ( $>12 \%)$ and nickel $(-8 \%)$ are commonly added to stainless steel to provide corrosion and oxidation resistance. On contact with oxygen, a chromium oxide layer is formed on the surface of the stainless steel. This passive layer is able to protect the underlying material from corrosion. As a consequence, the oxygen content in the 
investigated stainless steel textiles, takes account of both (undesired) iron oxides and chromium oxide passivation layer.

The air permeability tests along with the EDS analysis,are useful tools for the interpretation of the infrared emissivity measured values, as shown in the last section.

\section{EXPERIMENTAL}

Among the direct radiometric emissivity measurements, quantitative characterization of the infrared radiation emitted by an object at a given temperature, also known as infrared thermography, can be obtained using a calibrated IR-camera (i.e. radiometric camera). As already mentioned, the infrared emissivity of the steel textiles was measured placing the textile sample in direct contact with the heat plate. A hotplate holder was employed, acting as the heat source, allowing maximum heating temperature $+600{ }^{\circ} \mathrm{C}$ with fast heating-up by powerful integrated electrical heater, homogenous temperature distribution and over-temperature protection inside the plate. A clear analogical display for setting of temperature of the integrated heater allows to set the temperature with a resolution of $\sim 20^{\circ} \mathrm{C}$. However, once the hotplate temperature is set with this resolution, the actual temperature is accurately read by a thermocouple, which is placed in direct contact with the heating plate. In order to avoid undesired oscillation of the heating current, a stabilized power supply was employed. A radiometric forward looking infrared camera operating in the mid-infrared wavelength range (COX320) was used to measure the amount of infrared radiation emitted by the different samples between $8 \mu \mathrm{m}$ and $14 \mu \mathrm{m}$, providing detailed thermographic images. The FPA sensor of this radiometric imaging system is based on a grid of $384 \times 288$ pixels, composed by uncooled A-Si microbolometer with pixel pitch of $25 \mu \mathrm{m}$ (spatial resolution) and a thermal sensitivity (or noise equivalent temperature difference, NETD) of $60 \mathrm{mK}$ at $300 \mathrm{~K}$. The microbolometers present a spectral sensitivity response which is almost flat over the wavelength range $8 \div 14 \mu \mathrm{m}$ included within the minimum and maximum value of $\sim 0.9$ and 1 , 
respectively. In order to prevent detector saturation the standard calibration range of the infrared camera $\left(-20^{\circ} \mathrm{C}\right.$ to $120^{\circ} \mathrm{C}$ ) was extended to high temperature detection mode (up to $650^{\circ} \mathrm{C}$ ), thus the temperature of sample holder could be increased up to $300^{\circ} \mathrm{C}$. The infrared camera system was integrated with a manual focus lens, having focal length of $40 \mathrm{~cm}$. Details of the experimental setup are shown in Figure 2.

In order to prevent spurious thermal reflections on the sample surface, generated by external environmental sources, the experimental apparatus was protected by black opaque shields, thus confining the complete camera field of view (FOV).

The standard test methods for measuring and compensating for emissivity using infrared imaging radiometers (ASTM E1933-99a), cover procedures for measuring emissivity of a specimen surface using infrared thermography [17]. They include contact (using a contact thermometer) and non-contact (using a surface modifying material -spray or paint- of known emissivity) methods [17]. Specifically, in our laboratory we implemented a contact-thermometer setup for measuring the emissivity of the investigated textiles.

For each set of experimental measurements, the steel textiles were kept in direct contact with the heating plate. According to this method, first of all, the infrared camera was pointed at the specimen and focused on the portion where the emissivity was to be measured. An appropriate infrared imaging radiometer measurement function, such as area temperature, was employed to average and measure sample temperature. Afterwards, the contact thermometer, which in our case is thermocouple Type T (copper-constantan), was employed to measure the temperature of the same area of the sample. Then, without moving the image, the computer's emissivity control was adjusted until thermographic camera indicates the same temperature as recorded with the contact thermometer. The indicated emissivity is thus the measured emissivity of the specimen at the investigated temperature, at the operational range of 
the infrared camera. The described procedure was repeated three times and then averaged to get an average emissivity value, for each temperature point.

This method requires the specimen to be at a temperature that is at least $10^{\circ} \mathrm{C}$ warmer or cooler than the ambient temperature. Potential errors can be minimized by ensuring the stability of the temperature difference between the specimen and the ambient temperature during the test. Also the emissivity measurement accuracy can be increased by increasing the mentioned temperature difference. Furthermore, the calibrated infrared camera along with its built-in software allows the operator to input ambient temperature incident upon the specimen, in order to compensate the reflected infrared radiation.

\section{RESULTS AND DISCUSSIONS}

The obtained infrared emissivity measured as a function of temperature are plotted (with symbols) in Figure 3, along with the corresponding fitting curves (solid lines, as described later). According to Stefan-Boltzmann's law, emissivity and the infrared emitted radiation (signal) are linked through the fourth power of temperature. We thus evaluated an error of $\pm 8 \%$ for the emissivity measurement, since temperature accuracy of $\pm 2 \%$ is reported in the technical specifications of the infrared camera.

The infrared emissivity properties of the investigated metallic textiles, were studied by means of the finite-difference time-domain (FDTD) numerical simulations, using a commercial software (Lumerical FDTD solutions). The spectral absorbance has been simulated, being the absorbance proportional to the emissivity. The simulations were performed over a computational domain of $(180 \times 180 \times 140)$ micron $^{3}$.For the first simulations the metallic textiles were modeled according to the scheme reported in Figure 4a.In the FDTD cell the internal core of the metallic wires are represented by four metallic (iron) cylinders having diameter of 140 micron disposed at the edge of a square $(180 \times 180$ micron $^{2}$ ), thus leaving an internal hole of $(40 \times 40)$ micron $^{2}$. In order to simulate the metallic fibers, each core cylinder is covered with smaller iron cylinders, having diameter of 14 micron. Once the permittivity 
of iron was taken into account, the resulting structure exhibits the spectral properties shown in Figure $4 \mathrm{~b}$ (red curve). Over the investigated spectral range, transmittance shows a low value of 5\% while reflectance is as high as $90 \%$. According to Kirchoff law, the resulting emissivity is proportional to the absorbance value, given by $\mathrm{A}=100-\mathrm{R}-\mathrm{T}=5 \%$. It's worth to note that if the structure is only formed by metallic cylinders, the obtained value is very low if compared with the experimental data.

In order to increase emissivity value, it was necessary to add some material which absorbs in the investigated range. Considering the possible occurrence of steel textile oxidation, we thus improved the design of the FDTD cell by adding some small cylinders of $\mathrm{FeO}_{2}$, having diameter of 4 microns, in order to reproduce the potential effect of distributed iron oxide on the overall spectral properties. $\mathrm{FeO}_{2}$ cylinders were thus disposed over the two crossed direction as shown in Figure 4c, with red colored cylinders. Given the dielectric constants of $\mathrm{FeO}_{2}[18]$ numerical simulations show that reflectance value strongly decreases at 10 micron $(\mathrm{R}=53 \%)$, while transmittance still preserves a low value of $3 \%$, producing an absorbance peak of about $A=44 \%$ (Figure $4 \mathrm{~b}$, blue curve). This numerical result points out that in order to increase the emissivity values, the addition of absorbing elements within the textile texture should be taken into account.

Given these considerations, in order to reconstruct the experimental data we employed Plank's law in the investigated temperature range, and retrieved the spectral dependence of emissivity, using a nonlinear least square fitting procedure. The presence of two resonances in the absorption coefficient of $\mathrm{FeO}_{2}$, within the investigated infrared range [18], suggested the use of two Gaussian oscillators characterized by three different sets of parameters, i.e. the intensity factor (amplitude, $A$ ), the resonance frequency (center wavelength, $B$ ), and the broadening of absorption peak (line width, $C$ ) respectively. Neglecting terms in the visible, we assumed a spectral emissivity $\epsilon(\lambda)$ in the infrared camera operational range $(7 \div 15$ micron) having this form: 
$\varepsilon(\lambda)=\sum_{i=1}^{2} A_{i} e^{-\left(\frac{\lambda-B_{i}}{C_{i}}\right)^{2}} ;$

Specifically, the implemented least square fitting procedure employs the experimental input data (emissivity vs temperature) and retrieves a spectral emissivity in the operational wavelength range of the infrared camera, i.e. between $\lambda_{\min }$ and $\lambda_{\max }$, having a form given by Equation (1). The resulting spectral emissivity minimizes the difference between experimental data and the theoretical fit obtained using the following expression:

$$
\varepsilon(T)=\frac{\int_{\lambda_{\min }}^{\lambda_{\max }}\left(\frac{2 h c^{2} \varepsilon(\lambda)}{\lambda^{5}\left(e^{\left(\frac{h c}{K_{B} \lambda T}\right)}-1\right)}\right) d \lambda}{\int_{\lambda_{\min }}^{\lambda_{\max }}\left(\frac{2 h c^{2}}{\lambda^{5}\left(e^{\left(\frac{h c}{K_{B} \lambda T}\right)}\right)}\right) d \lambda} ;
$$

Where $h$ is the Planck constant, $c$ the speed of light in the medium and $K_{B}$ the Boltzmann constant, being $\epsilon(\lambda)$ the emissivity as a function of the wavelength.

Within the investigated temperature range, different emissivity values were found, standing for different degrees of absorption in the four investigated textiles, as reported in Table I. We show in Figure 5 the corresponding spectral emissivity curves obtained for the four samples. The area beneath each curve, i.e. the definite integral calculated between the two boundary wavelengths, is evidently proportional to the emissivity value in the investigated wavelength range. Furthermore, we added some arrows to show some characteristic absorption bands, corresponding to the prominent peaks of typical oxygen atom vibrational modes for metal oxide structures [19-20]. It's worth to note that those wavelengths where the emissivity equals to 1 are associated to a refractive index approaching unity 
value [18], thus resulting in negligible reflectivity, i.e. similarly to the so called Christiansen wavelength [21]. Following these considerations, we acknowledge that both iron and chromium oxide may display these absorption features, thus this fitting procedure is not intended to discriminate the nature of the oxide, but rather to provide an indication on the amount of the absorbing elements within the metallic textile.

Once the obtained spectral response $\epsilon(\lambda)$ is introduced into Equation (2), the temperature dependence $\epsilon(\mathrm{T})$ can be retrieved and the result of the fitting procedure is finally observable, and shown in Figure 3 with solid lines.

Metals typically show very high reflectance values in the IR range, thus for a metal-based textile, the higher the metal content, in terms of either thickness or metal composition, the lower the emissivity in the IR range. From the obtained results we can observe that non-woven and knitted textiles present high emissivity values within the investigated wavelength range. Considering the EDS data reported in Table II, the lowest chromium content (non-woven sample) as well as the lowest nickel content (knitted sample) make them more exposed to oxidation process. As a consequence, their absorbance comes out to be higher with respect to that of other investigated textiles. On the other hand, a reduced tendency to oxidation is reflected in the lower infrared emissivity measured for samples woven-1 and woven-2, both having high chromium and nickel weight percent (see Table II). Furthermore, air permeability tests, denote that woven fabric construction strengthens the thermal shielding performance. The low air permeability values (see Table I) obtained for woven-1 and woven-2 samples, in fact, imply more metallic yarns per $\mathrm{cm}^{2}$ and thus more metal content per $\mathrm{cm}^{2}$.

\section{CONCLUSIONS}

In conclusion, we performed experimental characterization of metallic textiles composed by differently assembled stainless steel yarns. Quantitative characterization of the infrared emissivity was retrieved by 
infrared thermography (from the infrared images obtained using a calibrated focal plane array (FPA) IRcamera operating in the wavelength range $8 \div 14$ microns), by placing the sample in direct contact with a heat source (hot plate). Emissivity measurements, along with air permeability tests and EDS analysis of the specific textile, allow to evaluate the thermal shielding behavior of the specific textile. All the investigated stainless steel textiles, in fact, consent to achieve efficient thermal shielding of the underlying heat source, giving reduced apparent temperature (as read by the infrared camera) with respect to the hot plate they're put in contact with, due to their low emissivity values. As a potential application, the obtained results pave the way to the design of customized IR shielding/emitting textiles, by proper selection of the suitable fabric type and yarns' geometrical parameters.

\section{ACKNOWLEDGMENTS}

This work has been performed in the framework of the project "FISEDA" granted by Italian Ministry of Defence. Dr. Marco Centini and Dr. Ilaria Fratoddi are kindly acknowledged for helpful discussion and interesting comments. We also wish to thank Dr. Francesco Mura for performing the EDS analysis on the investigated samples. 


\section{FIGURES}
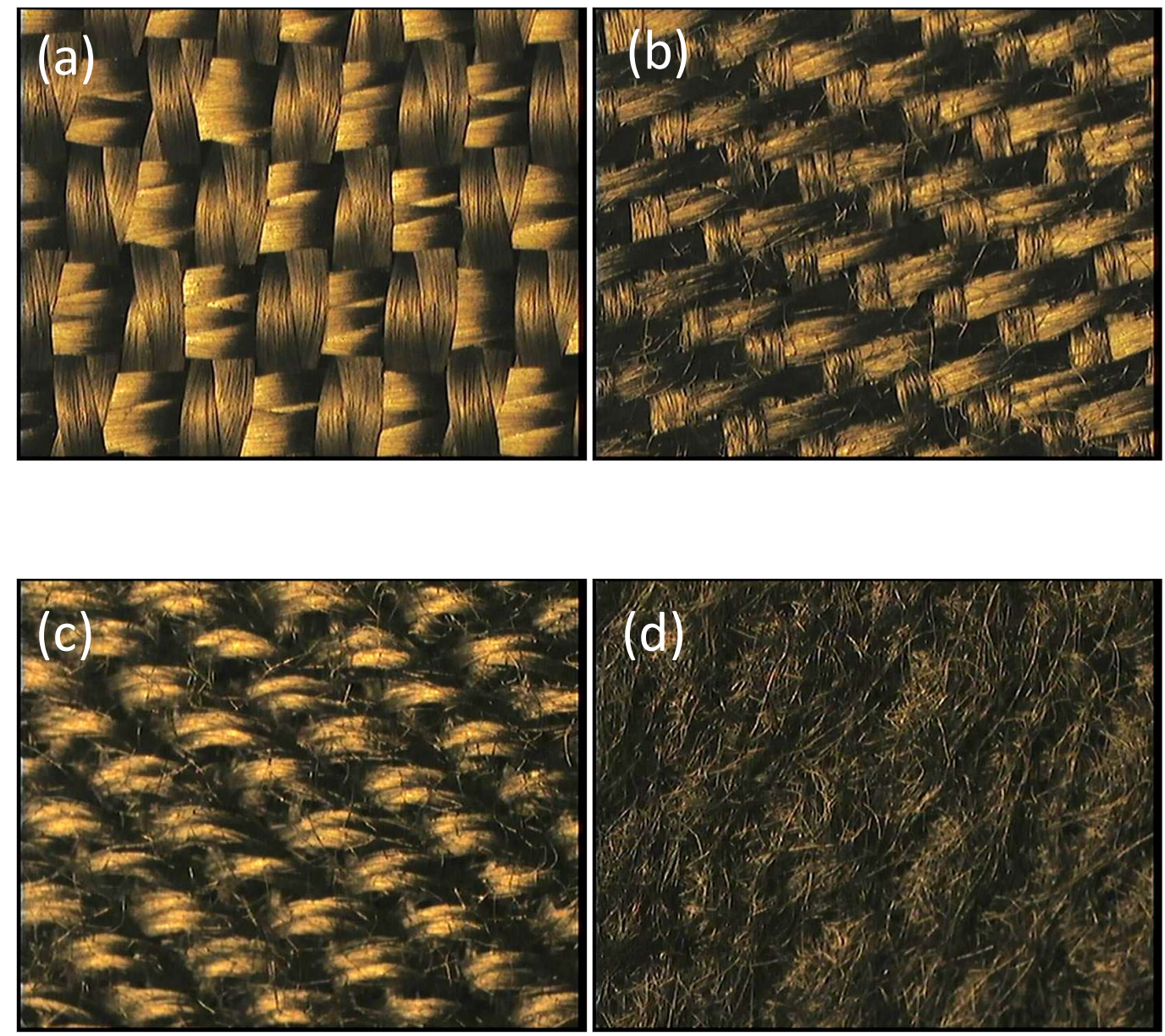

Figure 1. Digital images of the investigated textiles obtained by optical microscopy. In order to compare the different metallic fabrics, same magnification $(25 \times)$ was employed for all samples: (a) woven-1, (b) woven-2, (c) knitted, (d) non-woven (see Table I). 

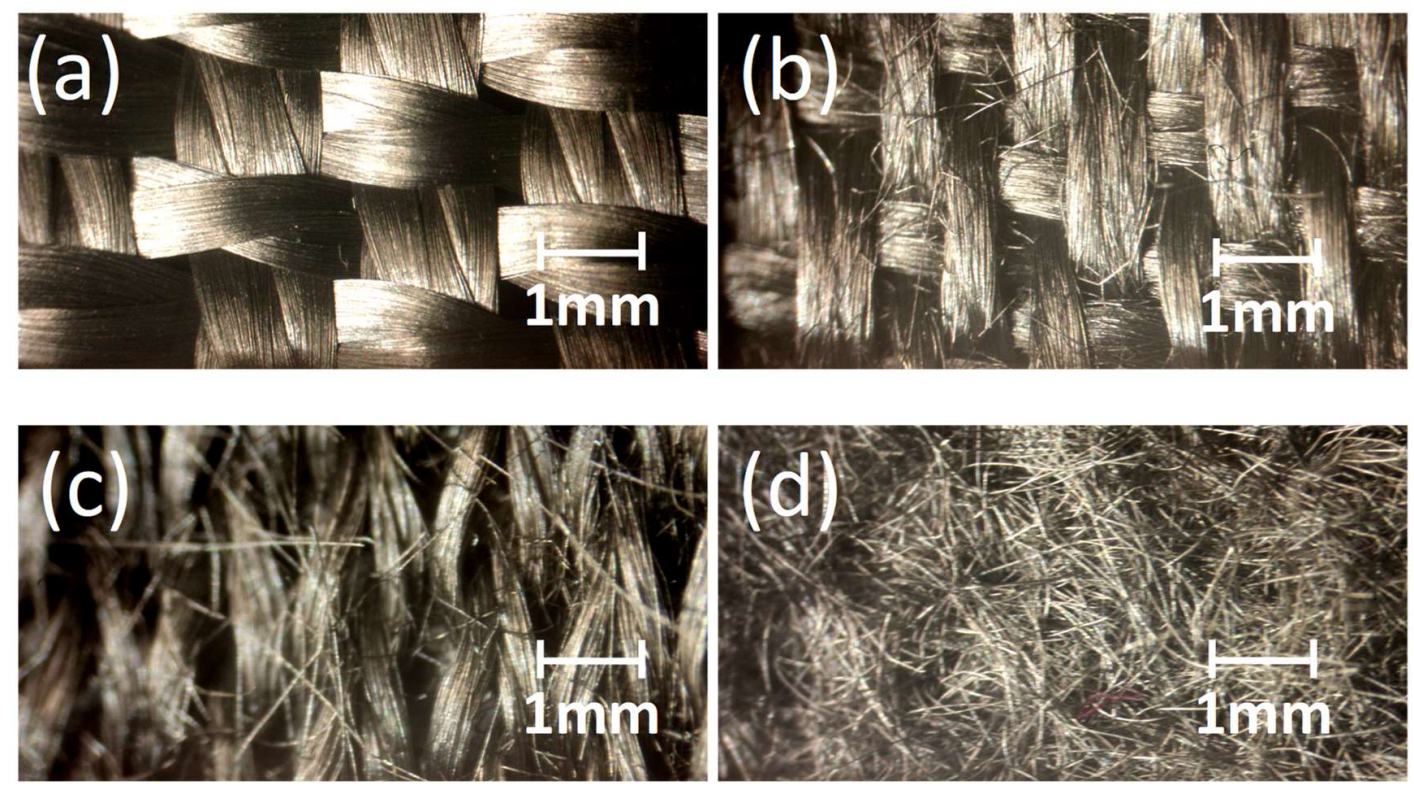

Figure 1. Digital images of the investigated textiles obtained by optical microscopy. In order to compare the different metallic fabrics, same magnification $(25 \times)$ was employed for all samples: (a) woven-1, (b) woven-2, (c) knitted, (d) non-woven (see Table I). 


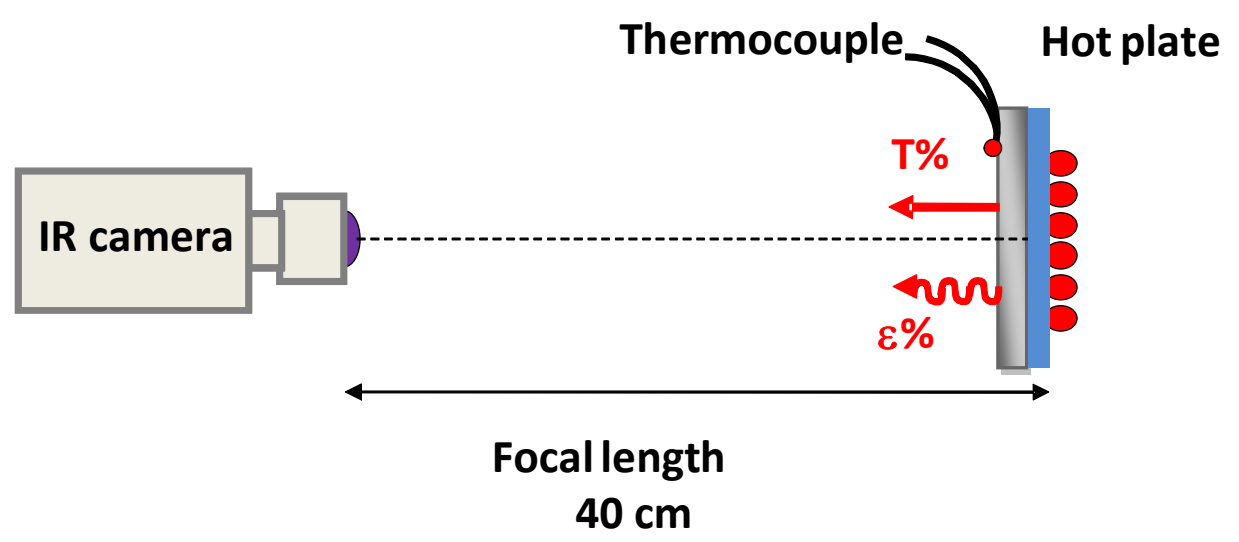

Figure 2. Schematic drawing of the infrared thermography experimental setup. 


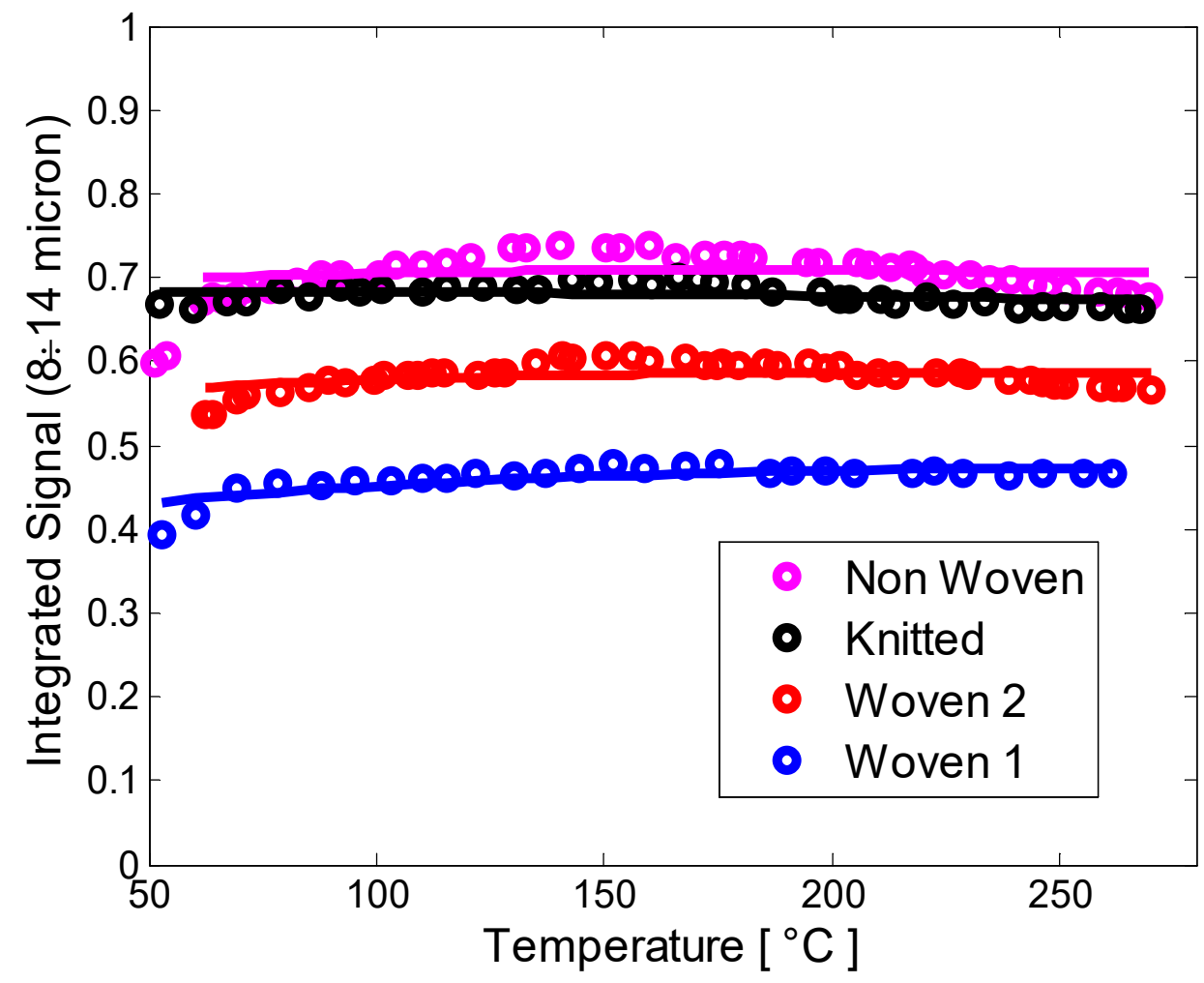

Figure 3. Experimental plot of the emissivity as a function of temperature (symbols) measured in the LWIR range, i.e. $8 \div 14$ micron, for all the investigated samples (see legend). The continuous lines represent the fitting curves obtained using Equation (2). 

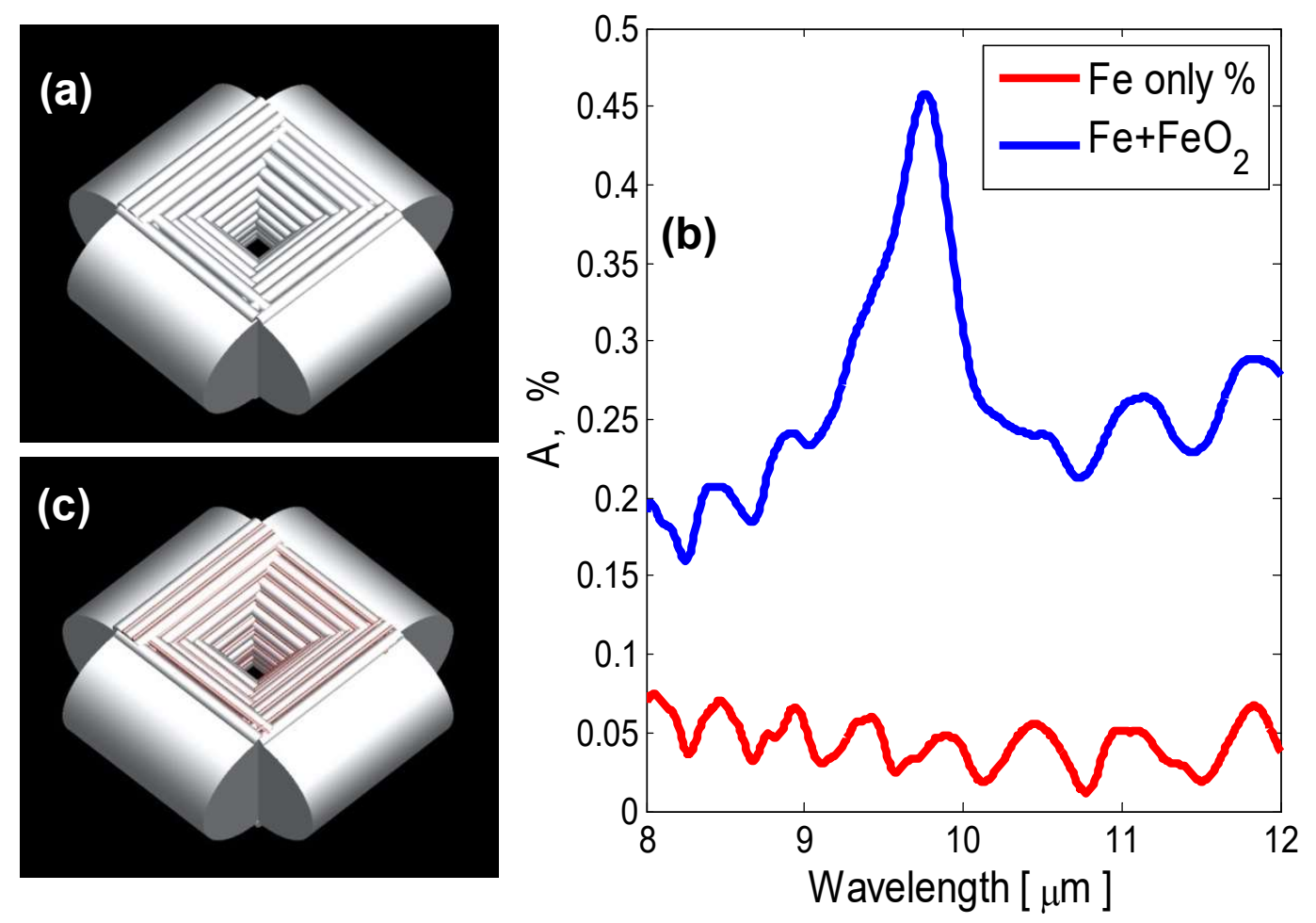

Figure 4. Scheme of the investigated metallic textiles, as modeled by means of the finite-difference timedomain (FDTD) numerical simulations: (a) metallic (iron) yarns; (b) spectral absorbance calculated for iron yarns (red curve) and iron/iron oxide yarns, respectively; (c) metallic yarns covered with $\mathrm{FeO}_{2}$ cylinders (in red). 


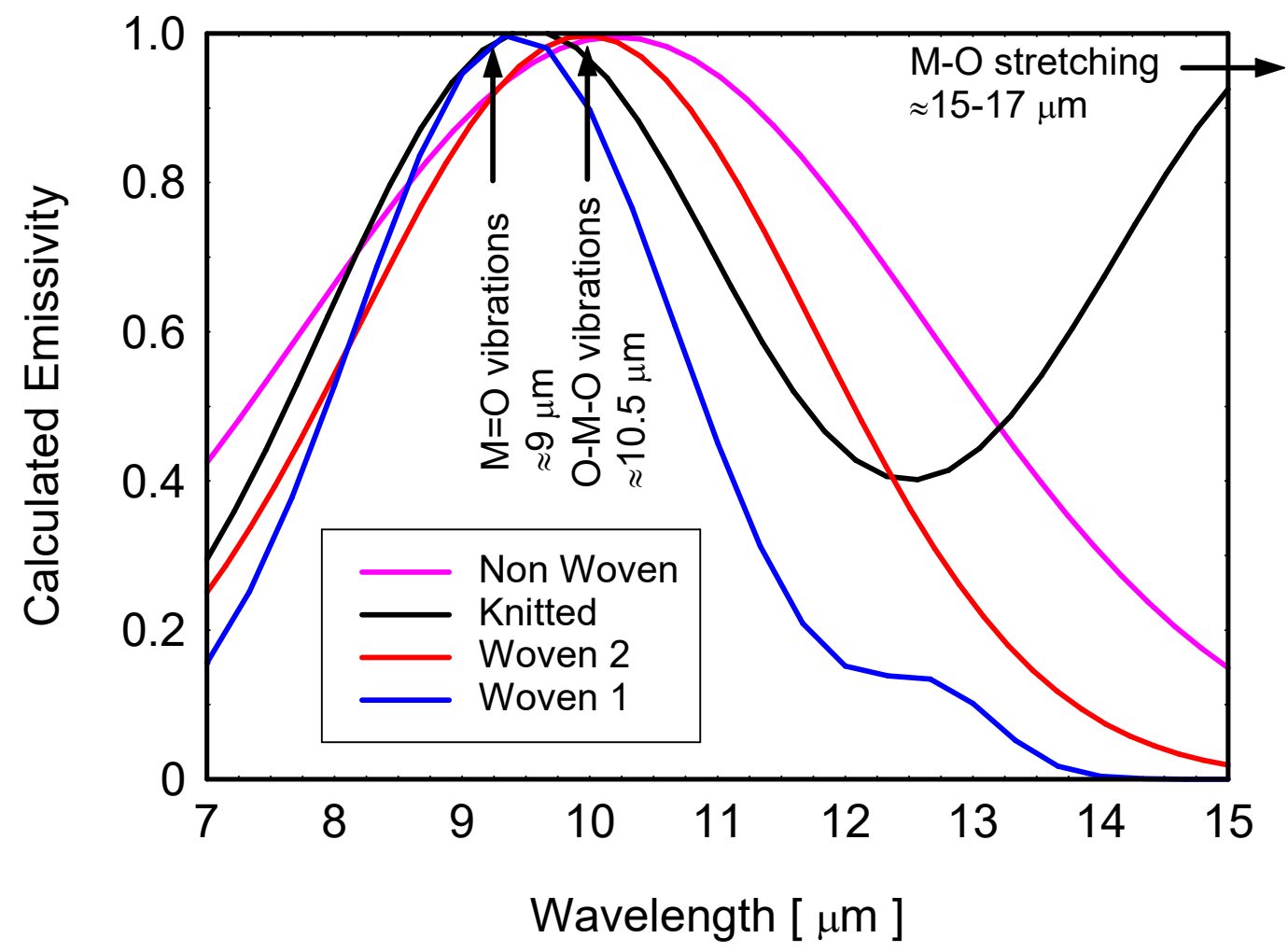

Figure 5.The spectral emissivity curves of the investigated samples (see legend) having a form given by Equation (1), as retrieved using a least square fitting procedure. 
Table I. Experimental (average measured emissivity, air permeability) and theoretically calculated data (emissivity as a function of temperature $\epsilon(\mathrm{T})$, and oscillator constants of spectral emissivity $\epsilon(\lambda)$ ), resulting from stainless steel textiles characterization:

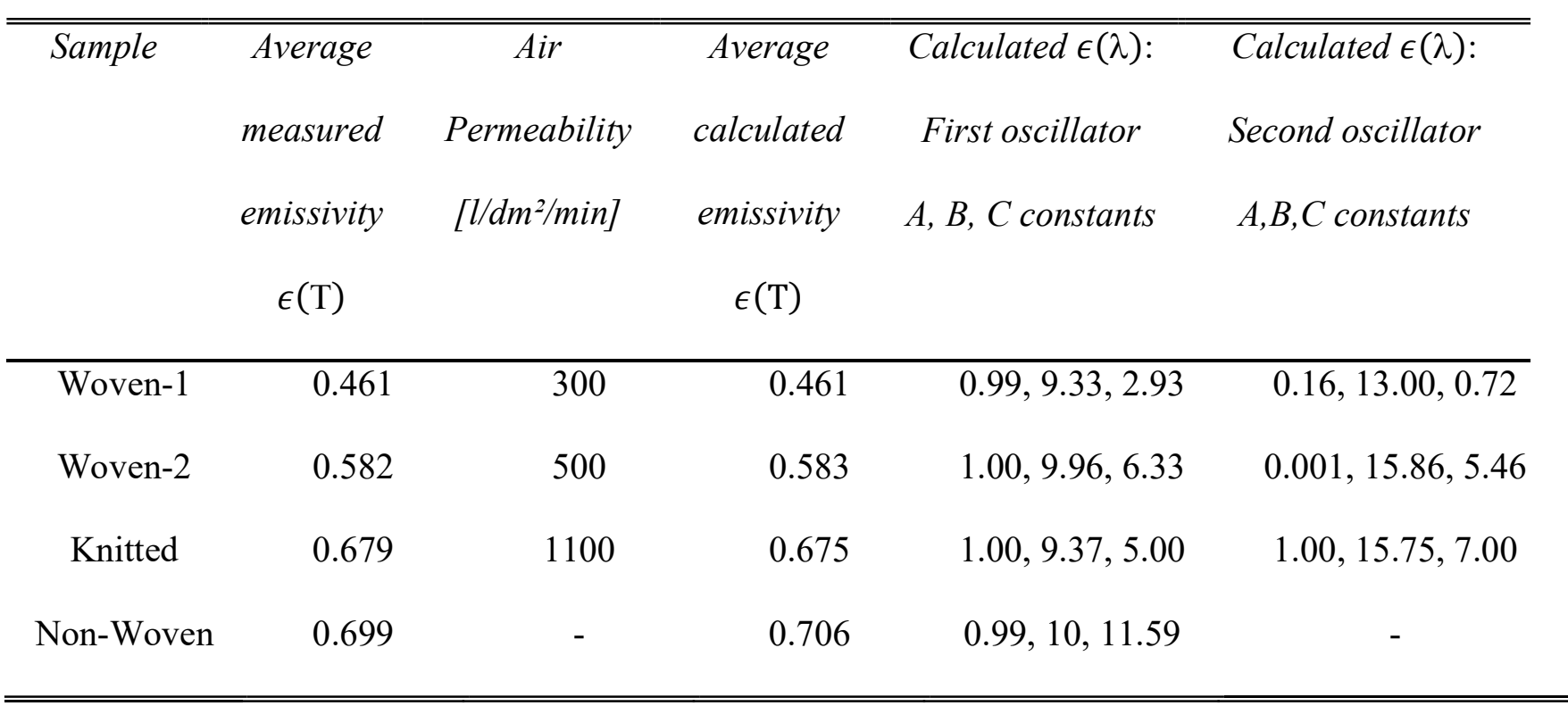

Table II. Elemental analysis of the investigated stainless steel textiles obtained from Energy Dispersive X-ray Spectroscopy (SEM/EDS).

\begin{tabular}{ccccc}
\hline \hline Sample & Owt\% & Fe wt\% & Cr wt\% & Ni wt\% \\
\hline Woven-1 & 0 & 67.50 & 17.37 & 9.71 \\
Woven-2 & 1.23 & 65.96 & 18.08 & 8.18 \\
Knitted & 0.70 & 74.93 & 17.21 & 3.95 \\
Non-Woven & 1.38 & 69.95 & 13.86 & 9.82 \\
\hline \hline
\end{tabular}




\section{REFERENCES}

[1] F. Soldani, "Infrared signature: Theory and example of practical measurement methods", 2nd IEEE International Workshop on Metrology for Aerospace, MetroAeroSpace 2015 -Proceedings 7180703, 472-478 (2015).

[2] F. Liu, X. Shao, P. Han, B. Xiangli and C. Yang, "Detection of infrared stealth aircraft through their multispectral signatures”, Optical Engineering 53, 094101 (2014).

[3] G.A. Rao and S.P. Mahulikar, "Effect of atmospheric transmission and radiance on aircraft infrared signatures”, Journal of Aircraft 42, 1046-1054 (2005).

[4] M.C. Larciprete, A. Belardini, R. Li Voti and C. Sibilia, "Pre-fractal multilayer structure for polarization- insensitive temporally and spatially coherent thermal emitter", Optics Express 21, A576A584 (2013).

[5] M.C. Larciprete, M. Centini, R. Li Voti, M. Bertolotti and C. Sibilia, "Polarization insensitive infrared absorbing behaviour of one-dimensional multilayer stack: a fractal approach", Optics Express 22 (S6), A1547-A1552 (2014).

[6] M.C. Larciprete, C. Sibilia, S. Paoloni, G. Leahu, R. Li Voti, M. Bertolotti, M. Scalora and K. Panajotov, "Thermally induced transmission variations in $\mathrm{ZnSe} / \mathrm{MgF}_{2}$ photonic band gap structures", Journal of Applied Physics 92, 2251-2255 (2002).

[7] R. Li Voti, M.C. Larciprete, G. Leahu, C. Sibilia and M. Bertolotti. “Optimization of thermochromic $\mathrm{VO}_{2}$ based structures with tunable thermal emissivity", Journal of Applied Physics 112,034305 (2012).

[8] O.B. Wright, R. Li Voti, O. Matsuda, M.C. Larciprete, C. Sibilia and M. Bertolotti, "Photothermal probing of inhomogeneously modulated transparent thin films", Journal of Applied Physics, 91, 50025009 (2002). 
[9] M.C. Larciprete, A. Albertoni, A. Belardini, G. Leahu, R. Li Voti, F. Mura, C. Sibilia, I. Nefedov, I.V.Anoshkin, E.I. Kauppinenand A.G. Nasibulin, "Infrared properties of randomly oriented silver nanowires", Journal of Applied Physics 112, 083503 (2012).

[10] R. Li Voti, G. Leahu, M.C. Larciprete, C. Sibilia, M. Bertolotti, I. Nefedov and I.V. Anoshkin, "Photoacoustic Characterization of Randomly Oriented Silver Nanowire Films", International Journal of Thermophysics 36, 1342-1348 (2015).

[11] K. Fortuniak, G. Redlich, E. Obersztyn, M.Olejnik, A. Bartczak and I. Król, "Assessment and Verification of the Functionality of New, Multi-Component, Camouflage Materials", Fibres\& Textiles in Eastern Europe 101, 73-79 (2013).

[12] G. Redlich, E. Obersztyn, M. Olejnik, K. Fortuniak, A. Bartczak, L. Szugajew and J. Jarzemski, "New Textiles Designed for Anti-Radar Camouflage", Fibres\& Textiles in Eastern Europe 103, 34-42 (2014).

[13] A. Bedeloglu, "Investigation of electrical, electromagnetic shielding, and usage properties of woven fabrics made from different hybrid yarns containing stainless steel wires", The Journal of the textile institute 104, 1359-1373 (2013).

[14] L. Cheng, T. Zhang, M. Guo, J. Li, S. Wang and H. Tang, "Electromagnetic shielding effectiveness and mathematical model of stainless composite fabric", The Journal of the textile institute 106, 577-586, (2015).

[15] V. Rubežienè, I. Padleckienè, S. Varnaitè-Žuravliova and J. Baltušnikaite, "Reduction of Thermal Signature Using Fabrics with Conductive Additives”, Materials Science (Medžiagotyra)19, 409-414 (2013).

[16] Jung-Sim Roh, Yong-Seung Chi and Tae Jin Kang, "Thermal insulation properties of multifunctional metal composite fabrics”, Smart Materials and Structures 18, 025018 (2009). 
[17] ASTM E1933-99a, "Standard Test Methods for Measuring and Compensating for Emissivity Using Infrared Imaging Radiometers", American Society for Testing and Materials International, West Conshohocken, PA (1999).

[18] T.D. Glotch and G.R. Rossman, "Mid-infrared reflectance spectra and optical constants of six iron oxide/oxyhydroxide phases", Icarus 204, 663-671 (2009).

[19] Metal Oxide Catalysis, Volume 1, Edited by S. David Jackson and Justin S. J. Hargreaves, WileyVCH Verlag GmbH \& Co. KGaA, Weinheim, Germany (2009).

[20] E.L. Lee and I.E. Wachs, "In situ spectroscopic investigation of the molecular and electronic structures of $\mathrm{SiO}_{2}$ supported surface metal oxides ", Journal of Physical Chemistry C 111, 14410-14425 (2007).

[21] B. Rousseau, J.F. Brun, D. De Sousa Meneses and P. Echegut, "Temperature measurement: Christiansen Wavelength and blackbody reference", International Journal of Thermophysics 26, 1277$1286(2005)$. 